\title{
Búsquedas relacionadas a actividad sexual en español: una aproximación usando Google Trends
}

\author{
Searches related to sexual activity in Spanish: an approach using Google Trends
}

\section{Sr. Editor:}

Aun cuando el ser humano no es procreador estacional, algunos estudios sugieren que la frecuencia con la que tiene relaciones sexuales seguiría ciertas fluctuaciones periódicas (1). Una forma de evaluar indirectamente este aspecto es analizar las búsquedas que se realizan en Google. Dos estudios realizados en Estados Unidos han encontrado fluctuaciones periódicas en las búsquedas de términos de contenido sexual (2) y de términos relacionados a la búsqueda de pareja sexual (3). Sin embargo, no hemos encontrado estudios similares en otros países.

Para evaluar la estacionalidad, se analizó las tendencias de búsquedas en internet, en español, que pudieran estar relacionadas a mayor actividad sexual, usando la herramienta Google Trends (www.google. com/trends) que permite estudiar las búsquedas realizadas en Google (4). Para ello, evaluamos términos de búsqueda posiblemente usados ante la proximidad de encuentros sexuales: ["sexo oral" + "sexo anal" + "posiciones sexuales" + "juguetes sexuales"]. Las tendencias de búsquedas fueron analizadas para cuatro países de habla hispana: dos del hemisferio sur (Perú y Chile), y dos del hemisferio norte (México y España), durante el periodo 2007-2017.

Para los países del hemisferio sur, observamos picos anuales en las búsquedas relacionadas a actividad sexual en los meses de diciembre-enero (Figura 1a y 1b), lo cual coincide con los hallazgos de un estudio realizado en Australia, donde se observó un incremento en el número de parejas sexuales durante los meses de verano (diciembre-febrero) (5). Para los países del hemisferio norte, observamos picos en los meses de junio-julio, y picos menores en los meses de diciembre-enero (Figura 1c y 1d), lo cual coincide con una revisión de la literatura que reportó mayor número de relaciones sexuales en Estados Unidos durante los meses de verano (junio a agosto) y de invierno (diciembre-enero) (1).

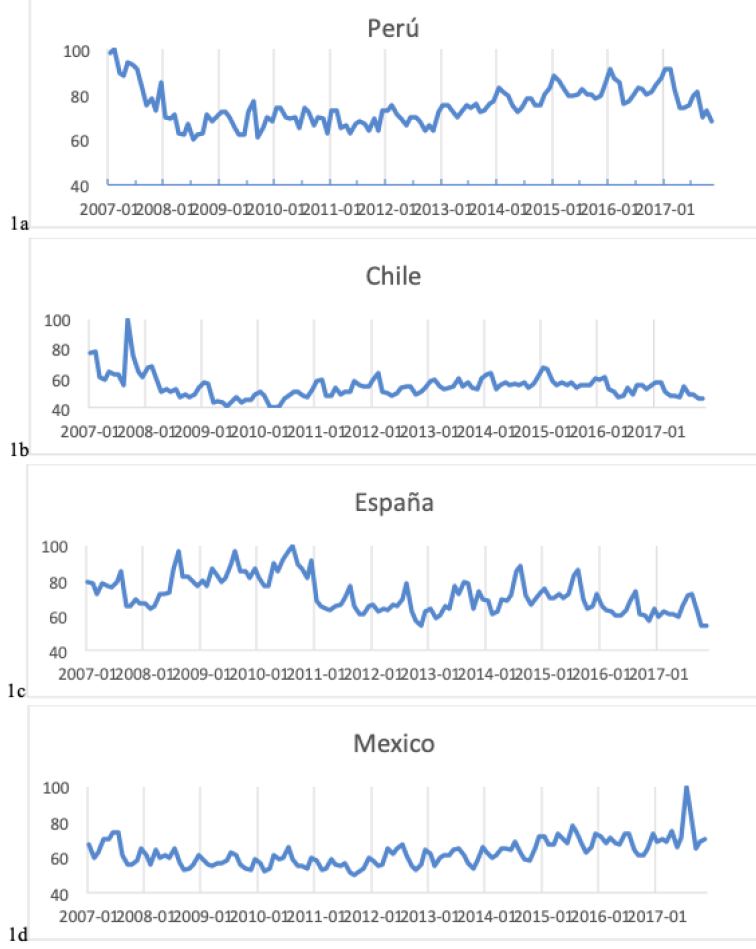

Figura 1. Tendencias en búsquedas relacionadas a actividad sexual, usando Google Trends*

*Las líneas verticales en cada figura señalan el mes de enero de cada año. 
Dado que los picos más pronunciados en ambos hemisferios coinciden con los meses de verano y con las vacaciones de verano, el aumento en las relaciones sexuales podría deberse al clima, a la mayor disponibilidad de tiempo, o al mayor tiempo sin supervisión (1).

Estas tendencias son importantes, pues las campañas de educación sobre métodos anticonceptivos y enfermedades de transmisión sexual pueden ser más provechosas si se realizan al comenzar los meses en los que la actividad sexual aumenta. Si bien las campañas internacionales más importantes sobre el tema (Día internacional de la planificación familiar: 3 de agosto, y Día Mundial de la lucha contra el SIDA: 1 de diciembre) coincidirían con los picos de actividad sexual en el hemisferio sur (diciembre-enero), no lo harían con los picos en el hemisferio norte (juniojulio). Por lo cual, de comprobarse esta estacionalidad, se podría plantear la realización de campañas en distintas fechas para cada hemisferio.

Finalmente, es necesario reconocer que los términos evaluados podrían no estar reflejando solamente tendencias de actividad sexual, sino también las tendencias de búsqueda de pornografía. Además, los resultados de Google Trends pueden estar influenciados por otros sucesos que aumenten el interés por los términos usados. Por ello, futuros estudios son necesarios para confirmar las tendencias aquí sugeridas.

\section{Correspondencia:}

Elizabeth Zavaleta-Lopez

Universidad Católica Los Ángeles de Chimbote

Instituto de investigación, Chimbote, Perú.

Dirección: Jirón Tumbes $N^{\circ}$ 247, Casco Urbano, Chimbote 43, Perú.

Correo: ezavaletal@uladech.edu.pe

Telf. +51934835272

\section{REFERENCIAS BIBLIOGRÁFICAS}

1. Macdowall W, Wellings K, Stephenson J, et al. Summer nights: A review of the evidence of seasonal variations in sexual health indicators among young people. Health Educ. 2007; 108(1):40-53. DOI: https://doi.org/10.1108/09654280810842120

2. MacInnis CC; Hodson G. Do American States with More Religious or Conservative Populations Search More for Sexual Content on Google? Arch Sex Behav. 2015;44(1):137-47. DOI: 10.1007/s10508-0140361-8

3. Markey P, Markey C. Seasonal variation in internet keyword searches: a proxy assessment of sex mating behaviors. Arch Sex Behav. 2013;42(4):515-521. DOI: $10.1007 / \mathrm{s} 10508-012-9996-5$

4. Google. Cómo se ajustan los datos de Trends - Ayuda de Tendencias de búsqueda; 2017 [Citado el 3 de abril del 2019]. Disponible en: https://support.google.com/ trends/answer $/ 4365533$ ? $\mathrm{hl}=$ es\&ref topic $=6248052$.

5. Cornelisse V, Chow E, Chen $\mathrm{M}$, Bradshaw C; Fairley C. Summer heat: a cross-sectional analysis of seasonal differences in sexual behaviour and sexually transmissible diseases in Melbourne, Australia. Sex Transm Infect. 2016; 92(4):286-91. DOI: 10.1136/sextrans-2015-052225

\section{Elizabeth Zavaleta-Lopez ${ }^{1, \text { a }}$ Jessica Hanae Zafra-Tanaka, ${ }^{2,}$, Alvaro Taype-Rondan ${ }^{3, b}$}

Recibido: 04/04/2019

\footnotetext{
Universidad Católica Los Ángeles de Chimbote, Instituto de investigación, Chimbote, Perú.

CRONICAS Centro de Excelencia en Enfermedades Crónicas, Universidad Peruana Cayetano Heredia, Lima, Perú.

Unidad de Investigación para la Generación y Síntesis de Evidencias en Salud, Universidad San Ignacio de Loyola, Lima, Perú.

Licenciada en Obstetricia;

Máster en ciencias de investigación epidemiológica.
} 